\title{
Diagnosis and Therapeutic Tactics of Landau-Kleffner Syndrome in Adults
}

\author{
Nadir A. Aliyev ${ }^{* *}$ and Zafar N. Aliyev ${ }^{2}$ \\ ${ }^{1}$ Department of Psychiatry and Addiction, Azerbaijan State Advanced Training Institute for Doctors named by A. Aliyev, Azerbaijan \\ ${ }^{2}$ Azerbaijan Medical University, Department of Psychiatry, Azerbaijan
}

${ }^{\star}$ Corresponding author: Nadir A. Aliyev, Department of Psychiatry and Addiction, Azerbaijan State Advanced Training Institute for Doctors named by A. Aliyev, Baku City, U. Chagibekov Street, Azerbaijan Republic; E-mail: aliyevnadir@yahoo.com

Received: July 07, 2021; Accepted: July 11, 2021; Published: July 15, 2021

\begin{abstract}
Purpose: The clinical signs of Landau-Kleffner syndrome (LCS) are electroencephalographic features and are discussed in five patients aged 25-33 years.

Methods: LCS diagnostic criteria: 1) acquired aphasia or oral hearing aphasia; 2) to have focal, tonic-clonic seizures. The patients were observed at the Mental Health Center of the Ministry of Health of the Republic of Azerbaijan. Of the five people examined, four were women.

Results: The mean follow-up lasted 24 months (January 2019 to January 2020) and tonic-clonic seizures were observed in all five patients. Oral auditory agnosia was found to be moderately aphasic in all patients. Typical epileptiform EEG patterns in SLK are high-amplitude (200-400 $\mu$ V) regional adhesions, sharp waves.

Conclusions: Landau-Kleffner syndrome is characterized by acquired oral hearing aphasia, seizures, and EEG changes in most patients. Patients were also prescribed depakine ${ }^{\circledR}$ chrono, lamotrigine and levetiracetam in combination with Cortexin ${ }^{\circledR}$. After 3 months of treatment, speech and cognitive dysfunction were prevented. Patients were monitored regularly for 24 years. Observations showed no convulsions or speech or cognitive impairment.
\end{abstract}

Keywords: LCS adults, Diagnostics, Treatment, Landau-Kleerner syndrome, Adult epilepsy, Treatment

\section{Introduction}

Landau-Kleffner syndrome (LCS) is a rare age-related epileptic encephalopathy characterized by developmental regression in the tongue and electroencephalogram (EEG) abnormalities located mainly around the temporo-parietal areas. If present, seizures consist of absence seizures or tonic-clonic episodes and are more likely to occur during sleep. Behavioral disorders can form part of the clinical picture. The syndrome described in 1957 [1] is also referred to as acquired aphasia with epilepsy (ICD-10, F80.3) to indicate the main features of this disease. It is considered a form of continuous burst during slow wave sleep (CSWS), although the two syndromes have different clinical manifestations and diagnostic implications.

The exact etiology of Landau-Kleffner syndrome is unknown. Structural brain damage in patients with LCS is very rarely associated with pathophysiology. Moreover, genetic factors may be involved. For example, the disease may be correlated with GRIN2A (16p13.2) mutations. This gene encodes a protein called GluN2A (also known as NR2A), which is a subunit of the glutamate ion channel receptor $\mathrm{N}$-methyl-D-aspartate (NMDA). It should be noted that NR2A can be identified at high concentrations in areas of the brain important for speech and language, while NMDA receptors are involved in a wide range of functions related to memory and learning. Again, GRIN2A changes correlate with a significant number of neurodevelopmental disorders, among which epilepsy may be a clinical manifestation [2,3].

It is difficult to express an estimate of the prevalence and incidence of the syndrome. Landau-Kleffner syndrome, indeed, is described as sporadic or limited case series. From the description of the syndrome, in 1957, no more than a few hundred cases have been reported in the literature. An epidemiologic study demonstrated that the incidence of children with LKS in Japan was about 1 in a million [4,5]. Furthermore, it emerges that males are more affected than females and that the reference age is between 3 and 8 years. However, documented cases also exist of younger children or adolescents with the syndrome.

We searched MEDLINE systems, - PreMEDLINE https://www. ncbi.nlm.nih.gov/pubmed/; https://www.ncbi.nlm.nih.gov/pmc/; The Cochrane Library; https://www.accessdata.fda.gov/scripts/ cder/daf/; http://www.ema.europa.eu/ema/; https://scholar.google. com; https://www.rxlist.com/script/main/hp.asp; http://www.nejm. org; https://www.bmj.com in order to find at least some information about the incidence of Landau-Kleffner syndrome in adults. The main task of the search was to find information about the presence of Landau-Kleffner syndrome in adults. In none of these search engines did we find data on the primary onset of Landau-Kleffner syndrome in adults. Based on the above, the aim of this study was to study the clinical picture and develop tactics for the treatment of LandauKleffner syndrome in adults. 


\section{Materials and Methods}

\section{Consent}

In accordance with the Helsinki Declaration of the World Medical Association "Recommendations for doctors engaged in bio-medical research involving people", adopted by the 18th World Medical Assembly (Finland, 1964, revised in Japan in 1975, Italy-1983, Hong Kong-1989, the South African Republic- 1996, Edinburgh-2000); The Constitution of the Republic of Azerbaijan, the Law "On Psychiatric Assistance" (adopted on 12.06.2001, with amendments and additions-11.11.2011. Parents or guardians have provided written informed consent to provide specific anonymized information obtained from their clinic visits for research use and have been reassured that their participation in the study is not related to ongoing clinical care. Consensus and data were obtained as patients were examined over a two-year period.

The decision of the Ethical Committee at the Azerbaijan Psychiatric Association on the article of NA. Aliev, ZN. Aliev "Clinical picture, diagnosis and therapeutic tactics of Landau-Kleffner syndrome in adults" submitted for publication in psychiatric journals: in connection with compliance with its legislative requirements and regulatory documents is to approve the article by NA. Aliyev, ZN. "Clinical picture, diagnosis and therapeutic tactics of Landau-Kleffner syndrome in adults" The patients were observed at the Mental Health Center of the Ministry of Health of the Republic of Azerbaijan from January 2018 to January 2020 for 24 months. Patients received depakin-chron $500 \mathrm{mg} 2$ times a day, lomotrigine $100 \mathrm{mg} 3$ times a day, levotisetam $1000 \mathrm{mg} 2$ times a day per os.

Additionally, the patients were assigned Cortexin ${ }^{\oplus}$ contains a complex of low-molecular water-soluble polypeptide fractions that penetrate through the BBB directly to nerve cells. The drug has a nootropic, neuroprotective, antioxidant and tissue-specific effect. The mechanism of action of the drug Cortexin ${ }^{\circledR}$ is due to the activation of peptides of neurons and neurotrophic factors of the brain; optimization of the balance of the metabolism of excitatory and inhibitory amino acids, dopamine, serotonin; GABAergic effects; a decrease in the level of paroxysmal convulsive activity of the brain, the ability to improve its bioelectrical activity; preventing the formation of free radicals (lipid peroxidation products). Active substance: polypeptides of cattle cerebral cortex. The drug is administered intramuscularly. Before injection, the contents of the vial are dissolved in $1 \mathrm{ml}$ of a $0.5 \%$ solution of procaine (novocaine), water for injection or $0.9 \%$ sodium chloride solution and injected once daily: adults at a dose of $10 \mathrm{mg}$ for 10 days; children from the neonatal period, with a body weight of up to $20 \mathrm{~kg}$ at a dose of 0.5 $\mathrm{mg} / \mathrm{kg}$, with a body weight of more than $20 \mathrm{~kg}$ - at a dose of $10 \mathrm{mg}$ for 10 days. Before injection, the contents of the vial are dissolved in $1 \mathrm{ml}$ of a $0.5 \%$ solution of procaine (novocaine), water for injection or $0.9 \%$ sodium chloride solution and injected once daily: adults at a dose of 10 $\mathrm{mg}$ for 10 days; If necessary, repeat the course in 3-6 months. Of the five people examined, four were women.

\section{$\underline{\text { Results }}$}

Routine neurological examination of patients, as a rule, does not reveal any focal symptoms. Magnetic resonance imaging (MRI) with
SLS, as a rule, does not reveal any pathology, however, it allows to exclude the symptomatic nature of the disease. The average follow-up lasted 24 months (January 2019 to January 2020) and tonic-clonic seizures were observed in all five patients. Oral auditory agnosia was found to be moderately aphasic in all patients. Typical epileptiform EEG patterns in SLK are high-amplitude (200-400 $\mu \mathrm{V})$ regional adhesions, sharp waves. Landau-Kleffner syndrome is characterized by acquired auditory aphasia, seizures, and EEG changes in most patients. Cortexin ${ }^{\circ}$ was also prescribed to patients with depakin-chrono, lamotrigine and levotirasem. After 3 months of treatment, speech and cognitive dysfunction were prevented. Patients were monitored regularly for 24 years. Observations showed no convulsions or speech or cognitive impairment.

\section{Discussion}

Our findings indicate that the most important characteristic of epileptiform activity in SLS is the tendency to diffuse spread. The diffuse propagation of peak-wave complexes in SLS is based on the phenomenon of secondary bilateral synchronization. At the same time, it is almost always possible to establish the temporal asynchrony of the complexes, as well as their amplitude predominance on the side dominant in speech. The highest amplitude is observed in the temporal leads, more often with an accent on the left; although cases of the location of the focus in the subdominant hemisphere of K.Yu. Mukhin [6] emphasize that sideliness of EEG disturbances does not always correspond to the dominant side, determined by the hand or eye, and epileptiform patterns can be observed in both the dominant and subdominant hemispheres [1].

This is the first preliminary descriptive study aimed at developing treatment strategies for clinical picture, diagnosis and therapeutic tactics of Landau-Kleffner syndrome in adults. It was found that Landau-Kleffner syndrome in adults after appropriate treatment, in all patients after appropriate treatment, in all patients, observations showed no convulsions or speech or cognitive impairment.

This study had several limitations; 1) a small number of patients; 2) a short time observation.

The sample size was relatively small. Further large-scale studies are needed to differentiate the use other anticonvulsants of LandauKleffner syndrome in adults.

\section{Conclusions}

Although clinical specimens are not representative of the wider population, this study nevertheless highlights the urgent need for further research of Landau-Kleffner syndrome in adults on the LCS diagnostic criteria-acquired aphasia or oral hearing aphasia, focal, tonic-clonic seizures. A. After appropriate treatment, in all patients, observations showed no convulsions or speech or cognitive impairment. The restoration of cognitive functions is very important to eliminate stigma and improve the quality of life of patients. It was found that in children the occurrence of aphasia not with epileptic seizures, but with epileptiform activity on the EEG, i.e., in fact, formulated the modern concept of epileptic encephalopathies. Our data indicate that in the Landau-Kleffner syndrome, the occurrence of aphasia is directly related to the frequency of epileptic seizures, 


\section{Author Disclosure}

Authors declare that the manuscript is submitted on behalf of all authors. None of the material in this manuscript has been published previously in any form and none of the material is currently under consideration for publication elsewhere other than noted in the cover letter to the editor. Authors declare to have any financial and personal relationship with other people or organizations that could inappropriately influence this work. All authors contributed to and have approved the final manuscript.

\section{Conflict of Interest Statement}

The authors declare that the research was conducted in the absence of any commercial or financial relationships that could be construed as a potential conflict of interest.

\section{References}

1. Landau WM, Kleffner FR (1957) Syndrome of acquired aphasia with convulsive disorder in children. Neurology 7: 523-530. [crossref]

2. Lesca G, Møller RS, Rudolf G, Hirsch E, Hjalgrim H, et al. (2019) Update on the genetics of the epilepsy-aphasia spectrum and role of GRIN2A mutations. Epileptic Disord 21: 41-47. [crossref]

3. Strehlow V, Heyne HO, Vlaskamp DRM, Marwick KFM, Rudolf G, et al. (2019) GRIN2A study group. GRIN2A-related disorders: genotype and functional consequence predict phenotype. Brain 142: 80-92. [crossref]

4. Kaga M, Inagaki M, Ohta R (2014) Epidemiological study of Landau-Kleffner syndrome (LKS) in Japan. Brain 36: 284-286. [crossref]

5. Caraballo RH, Cejas N, Chamorro N, Kaltenmeier MC, Fortini S, et al. (2014) Landau-Kleffner syndrome: a study of 29 patients. Seizure 23: 98-104. [crossref]

6. Mukhin K Yu (2016) Landau-Keffner syndrome (acquired epileptic. aphasia) with a focus on electroencephalographic criteria. Russian Journal of Child Neurology 11: 3 .

\section{Citation:}

